\title{
Adult Intussusception Caused by Inverted Meckel's Diverticulum Containing Mesenteric Heterotopic Pancreas and Smooth Muscle Bundles
}

\author{
Seungkoo Lee · Seong Whi Cho ${ }^{1}$ \\ Departments of Anatomic Pathology and 'Radiology, Kangwon National University Hospital, Kangwon National University School of Medicine, Chuncheon, Korea
}

\begin{abstract}
Adult intussusception represents $5 \%$ of all intussusceptions. ${ }^{1,2}$ Most of these cases are related to organic lesions. ${ }^{1}$ Infrequently, an inversion of Meckel's diverticulum (MD) into the lumen of the small bowel can cause intussusception. ${ }^{3-5}$ The incidence of intussusception due to an inverted MD accounts for about $4 \%$ of all cases of intussusceptions. ${ }^{1,4}$ Heterotopic pancreas (HP) is defined as pancreatic tissue located at aberrant sites that lacks anatomic and vascular continuity with the main pancreas. ${ }^{6-9}$ The most common location of HP is the proximal gastrointestinal tract. ${ }^{6,10}$ The mesenteric location of HP is rare, and only a few cases of HP in the mesentery of the small intestine have been reported in the literature. ${ }^{8,9}$ Here, we present a very rare case of adult ileoileal intussusception caused by an inverted MD containing mesenteric HP and smooth muscle bundles.
\end{abstract}

\section{CASE REPORT}

A 55-year-old man presented with intermittent abdominal pain and hematochezia of a 2-month duration. He also reported several episodes of melena-type bowel movements. His medical history was unremarkable except for an appendectomy 10 years prior. Blood tests revealed a hemoglobin level of $8.3 \mathrm{~g} / \mathrm{dL}$ (NL, $13.3-16.5 \mathrm{~g} / \mathrm{dL}$ ) and a hematocrit level of $23.8 \%$ (NL, 38.647.3\%). All other laboratory findings were unremarkable. An endoscopic study was unable to locate the site of the bleeding.

\section{Corresponding Author}

Seungkoo Lee, MD

Department of Anatomic Pathology, Kangwon National University Hospital, Kangwon National University School of Medicine, 1 Gangwondaehak-gil, Chuncheon 24341, Korea

Tel: +82-33-258-9172, Fax: +82-33-258-2475, E-mail: jsklee@kangwon.ac.kr

Received: May 9, 2016 Revised: June 8, 2016

Accepted: June 14, 2016
An abdominal and pelvic computed tomography (CT) scan showed a pedunculated intraluminal mass with central heterogeneous fat attenuation (Fig. 1). The patient underwent a minilaparotomy for segmental resection. Upon gross examination, the specimen consisted of a 15-cm segment of the small bowel with a small dimple in the anti-mesenteric aspect, and there was a pedunculated polypoid mass $(5.1 \times 3.0 \times 2.8 \mathrm{~cm})$ with a bulbousshaped head (Fig. 2A). The stalk of the mass was lined by unremarkable mucosa. The cut section of the mass revealed a nodular lesion within the adipose tissue sandwiched between the continuous lining of the proper muscle layers (Fig. 2B). Microscopic examination revealed that the bulbous tip lesion was covered by the full thickness of the intestinal wall and had deep ulcerations (Fig. 2C). The mucosa of the tip contained nondysplastic epithelial glands. Focal heterotopic antral-type gastric tissue was also present (Figs. 2C, 3A). Interestingly, ectopic pancreatic tissue and smooth muscle bundles were located within the entrapped mesenteric fat (Fig. 2C, D). The mesenteric heterotopic pancreatic tissues were composed of all elements typically found in normal pancreatic tissue (Heinrich type I), such as acini (Fig. 3B), islet cells (Fig. 3B), and ducts (Fig. 3C) and several discontinuous fascicles of smooth muscles (Fig. 3D). Based on the clinical and histopathologic findings, the pedunculated intraluminal mass was diagnosed as inverted MD with a mesenteric HP and displaced smooth muscle bundles. The patient had an uneventful postoperative recovery, with no further episodes of lower gastrointestinal hemorrhage during the follow-up period.

\section{DISCUSSION}

Adult intussusception differs from childhood intussusception in its presentation, causes, and management. ${ }^{1,2,7}$ The presenting 
symptoms of adult intussusception are often nonspecific. In our case, the patient developed only intermittent abdominal pain, melena-type bowel movements, and a few episodes of hematochezia. In adult intussusception, it is important to identify the leading point. Several pathologic conditions have been reported as the leading point in intussusception, such as inflammatory disease, neoplasm, adhesion, $\mathrm{MD},{ }^{1}$ and $\mathrm{HP}^{2}{ }^{2}$ In the present case, the CT scan revealed ileoileal intussusception associated with inverted MD (Fig. 1). Although most MD remains asymptom-

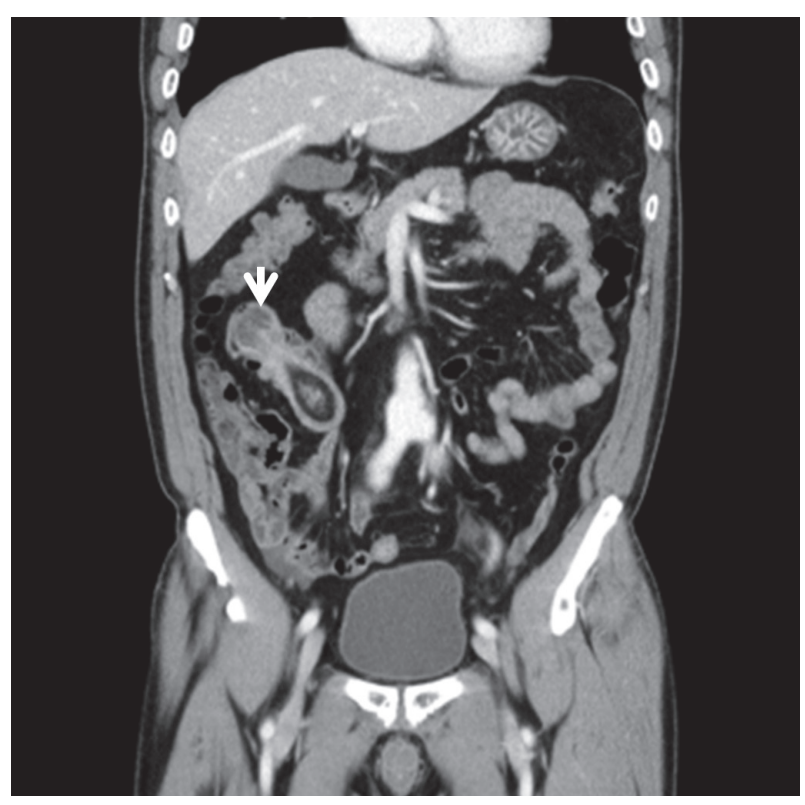

Fig. 1. Abdominal and pelvic computed tomography (CT) scan. The coronal view CT scan reveals an intestinal intussusception with an intraluminal mass (arrow). atic, it can be complicated by intussusception ${ }^{1,4,5,10}$ and gastrointestinal bleeding. ${ }^{10}$ Ulceration in MD is thought to occur mostly because of acid secretion by the heterotopic gastric mucosa. ${ }^{10}$ In the presented case, ulceration and gastrointestinal bleeding were associated with antral-type ectopic gastric tissue in the MD.

MD can invert into the intestinal lumen, but the exact mechanism of this inversion is not clearly understood. One theory is that abnormal peristaltic movement derived from the ulcer or ectopic tissue causes the diverticulum to become inverted. ${ }^{5}$ Once inverted, the diverticulum can serve as a site of intestinal obstruction or the leading point for an intussusception. In our case, the patient's inverted MD contained several aberrant displaced smooth muscle bundles (Fig. 2C, D). We propose that these aberrant smooth muscle bundles produced abnormal peristaltic movement.

HP is common in the upper gastrointestinal tract. ${ }^{6,8,9}$ Other possible locations include the gallbladder, bile duct, or omentum. ${ }^{6,9}$ In descending order of frequency, the involved histologic layers are the submucosa, ${ }^{1,5,6}$ muscularis propria, ${ }^{6}$ and serosa. ${ }^{6,7}$ HP located in the mesentery of the small intestine is very rare., In our case, the HP was found within the entrapped mesenteric fat tissue of the ileum. These displaced pancreatic tissues and aberrant smooth muscle bundles were associated with MD, which can act as a leading point for intussusception.

In summary, mesenteric HP and smooth muscle tissue can act clinically as a leading point for inverted MD and ileoileal intussusception.
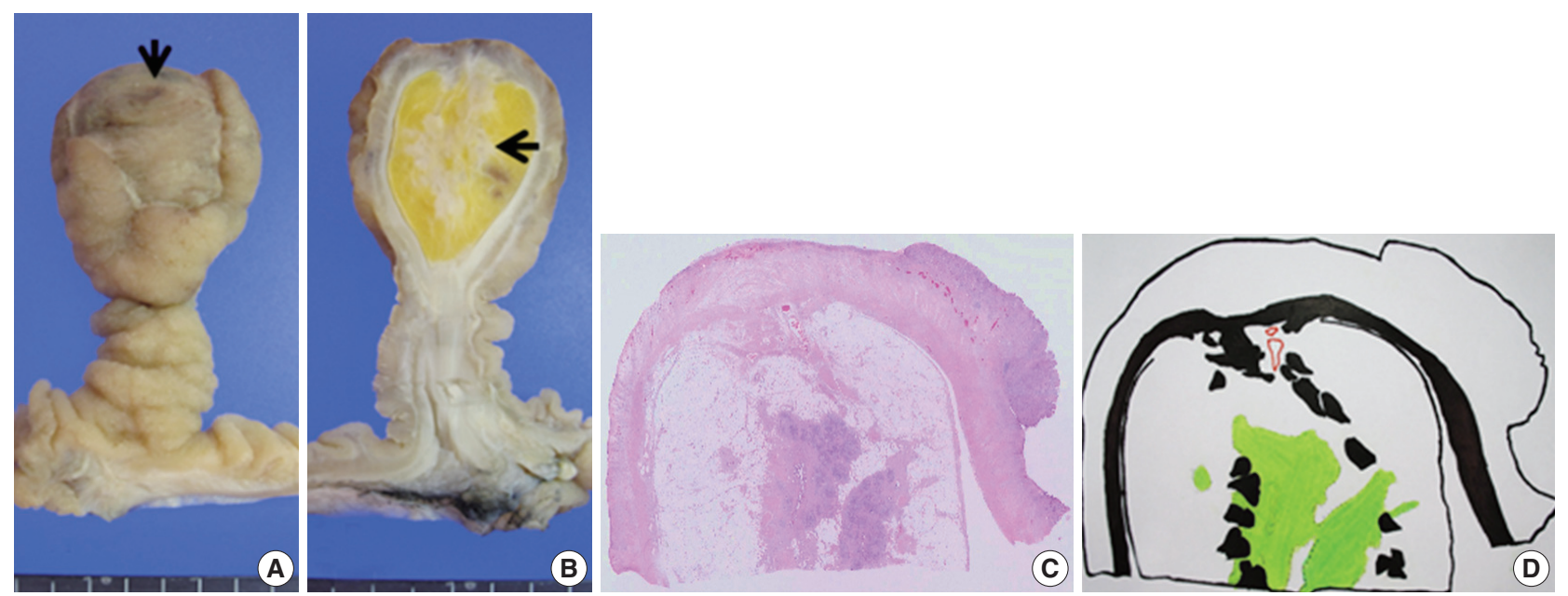

Fig. 2. The gross specimen and a schematic drawing of a hematoxylin and eosin (H\&E)-stained slide. The tip of the pedunculated polypoid mass shows a mucosal ulcer ( $A$, arrow) and mesenteric heterotopic pancreatic tissue (B, arrow). The entire image of the H\&E slide (C) and its schematic diagram (D) reveal mesenteric heterotopic pancreatic tissue (D, light green area) and smooth muscle tissue (D, black area). 

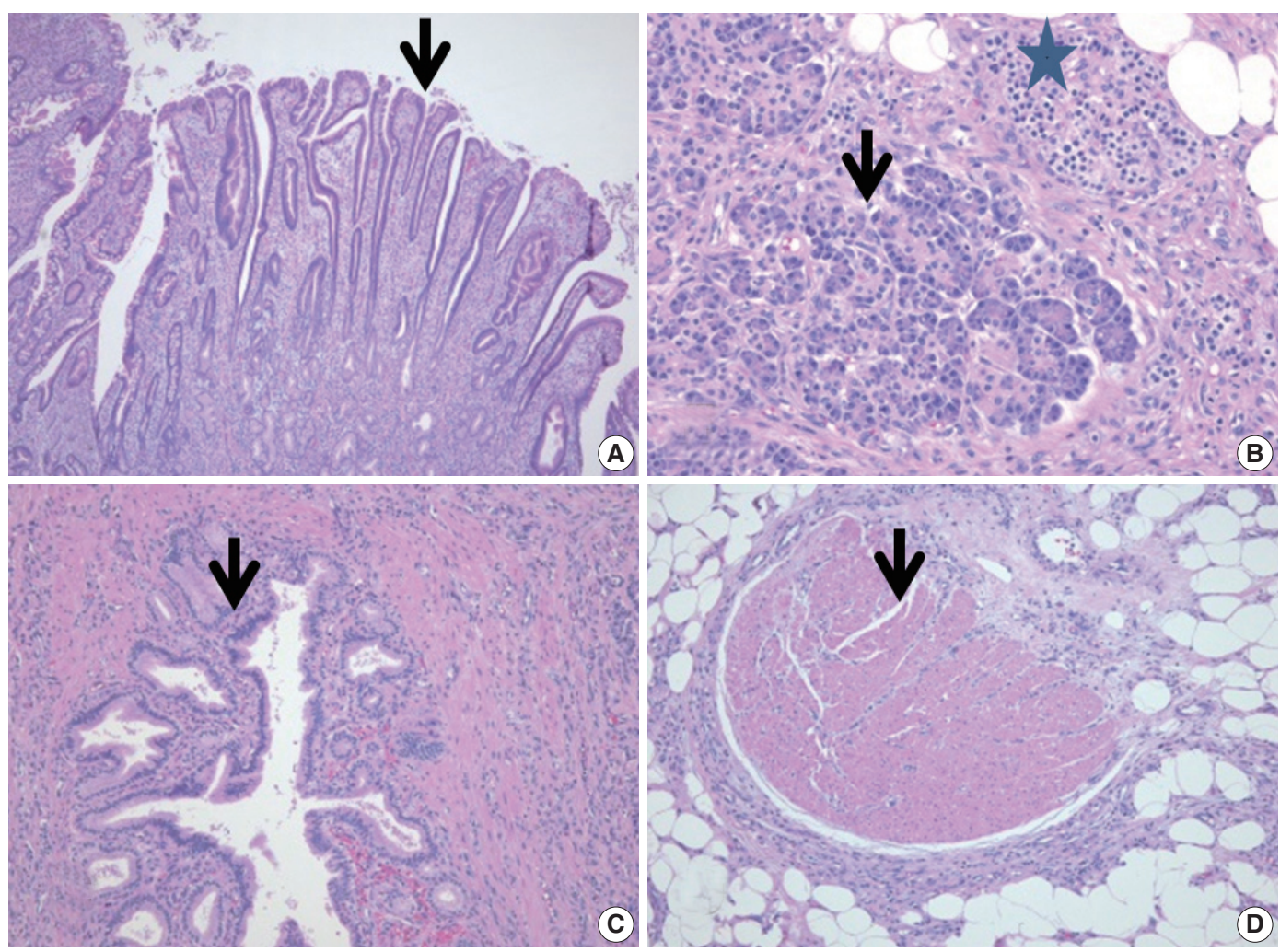

Fig. 3. Histologic features of the ectopic gastric mucosa and heterotopic pancreatic tissues. The inverted Meckel's diverticulum contains ectopic, antral-type gastric tissues ( $\mathrm{A}$, arrow) and mesenteric, heterotopic tissues such as acinar (B, arrow), islet (B, star), duct (C, arrow), and smooth muscle bundle (D, arrow).

\section{Conflicts of Interest}

No potential conflict of interest relevant to this article was reported.

\section{REFERENCES}

1. Ito T, Sato K, Maekawa H, et al. Adult intussusception caused by an inverted meckel diverticulum. Case Rep Gastroenterol 2011; 5: 320-4.

2. Chuang MT, Tsai KB, Ma CJ, Hsieh TJ. Ileoileal intussusception due to ileal ectopic pancreas with abundant fat tissue mimicking lipoma. Am J Surg 2010; 200: e25-7.

3. Levy AD, Hobbs CM. From the archives of the AFIP. Meckel diverticulum: radiologic features with pathologic correlation. Radiographics 2004; 24: 565-87.

4. Sioka E, Christodoulidis G, Garoufalis G, Zacharoulis D. Inverted Meckel's diverticulum manifested as adult intussusception: age does not matter. World J Gastrointest Surg 2011; 3: 123-7.
5. Kim SM, Jung MJ, Kim YO. Lipoma within Inverted Meckel's diverticulum: a case report. Korean J Pathol 2013; 47: 86-8.

6. Gupta MK, Karlitz JJ, Raines DL, Florman SS, Lopez FA. Clinical case of the month: heterotopic pancreas. J La State Med Soc 2010; 162: 310-3

7. Al-Zahem A, Arbuckle S, Cohen R. Combined ileal heterotopic pancreatic and gastric tissues causing ileocolic intussusception in an infant. Pediatr Surg Int 2006; 22: 297-9.

8. Canbaz H, Colak T, Dusmez Apa D, Sezgin O, Aydin S. An unusual cause of acute abdomen: mesenteric heterotopic pancreatitis causing confusion in clinical diagnosis. Turk J Gastroenterol 2009; 20: $142-5$.

9. Vyas S, Khandelwal A, Sandhu MS, Verma S, Khandelwal N. Asymptomatic heterotopic pancreas in the mesentery. Trop Gastroenterol 2012; 33: 289-92.

10. Kopacova M, Vykouril L, Vacek Z, et al. Inverted Meckel's diverticulum with ectopic pancreatic tissue as a source of severe gastrointestinal bleeding. J Gastrointest Surg 2010; 14: 578-81. 